\title{
Bi-conditional probabilistic fatigue stress-based curve definition and comparison with other models
}

\author{
Matteo Cova ${ }^{1,2, a}$, Marco Nanni ${ }^{2}$ and Roberto Tovo ${ }^{1}$ \\ ${ }^{1}$ Universita' di Ferrara, Dip. di Ingegneria, via Saragat 1, 44122 Ferrara, Italy \\ ${ }^{2}$ SACMI Imola S.C., via Selice 17/A, 40026 Imola (BO), Italy
}

\begin{abstract}
The definition of the relationship between probability, fatigue stress and cycles to failure is of great importance, especially in applications which requires very low probability of failure (e.g. $\mathrm{Pf}=0.1 \%$ ). In this paper a new formulation is presented, which allows to separately consider the probability of the endurance and the probability of existence of the initiating defect. This approach is then compared to a number of known models. For this purpose, the proprietary results of 8 fatigue test sets, each with at least 24 data points, have been analyzed. A ranking of goodness-of-fit based on the Relative Likelihood can be used to choose the best distribution within each model, but unfortunately cannot be used to compare different models. The conclusions were: 1 - the better description of the HCF strength, in terms of performance and robustness, were obtained by the 2-p Weibull distribution; 2- the choice of the model has a great influence in the estimate of the low probability quantiles, but it still subjective and a definitive answer cannot be given after this benchmark; 3- the strength of the proposed model is its flexibility.
\end{abstract}

\section{Introduction}

Components subjected to fatigue with a design endurance over $10^{7}$ and a feasible failure probability below $0.1 \%$ are the focus of this research. In essence, what is needed at the end is the fatigue strength distribution at the design endurance. Together with testing strategy, data analysis is crucial for deriving this information with confidence. In the following sections, a new model is presented and compared with 3 existing ones:

- Extended Stair-Case model (ESC) [1], in which all data (broken and run-outs) are treated as censored (left and right, respectively) and the chosen strength distribution is fitted using maximum likelihood. Normal, Log-normal and 2 and 3 parameter Weibull distributions were considered;

- Castillo Canteli model [2] defines an equivalent variable $v=f(S, N)$ which follows a 3-parameter Weibull distribution;

- Random Fatigue Limit model (RFL) [3] is a pretty sophisticated model in which both the distribution in strength and endurance can be log-normal or 2-p Weibull, and the distribution in endurance is heteroschedastic.

\footnotetext{
${ }^{a}$ Corresponding author: matteo. cova@sacmi.it
}

This is an Open Access article distributed under the terms of the Creative Commons Attribution License 4.0, which permits unrestricted use, distribution, and reproduction in any medium, provided the original work is properly cited. 


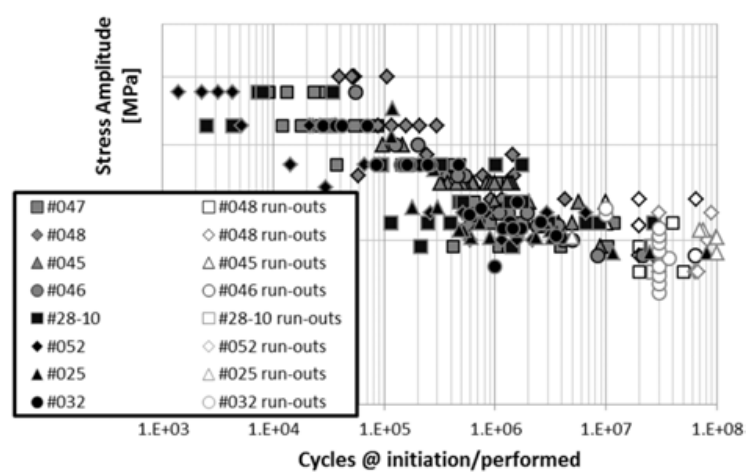

Figure 1. S-N plot of all the fatigue data.

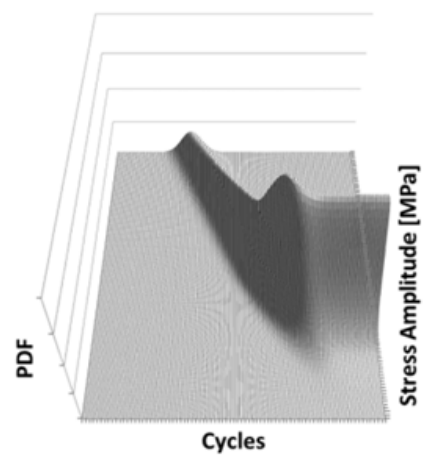

Figure 2. PDF of the Bi-Conditional fatigue curve.

8 proprietary fatigue tests provide the benchmark for the model comparison. The specimens were machined from material trepanned from heavy walled Ductile Iron castings.

\section{The Bi-conditional fatigue model}

The main basic concept is to separate the effect of a material defect to its existence. We combine the two effects by considering that the specimen under a fatigue stress $\overline{\sigma_{a}}$ will fail at $\bar{N}$ cycles if there is a defect "bad enough" to bring to failure under that stress AND the endurance is $\bar{N}$ In a statistic perspective, it is equal to consider the fatigue test as a parallel system consisting of two independent subsystems or events.

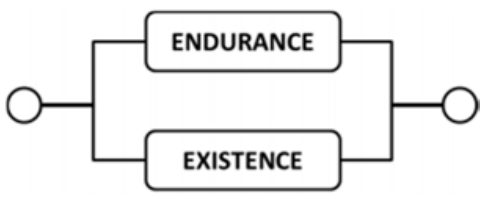

$$
P^{\text {bi-cond }}\left(\overline{\sigma_{a}}, \bar{N}\right)=P^{\text {endurance }}\left(\overline{\sigma_{a}}, \bar{N}\right) \cdot P^{\text {existence }}\left(\overline{\sigma_{a}}\right) \text {. }
$$




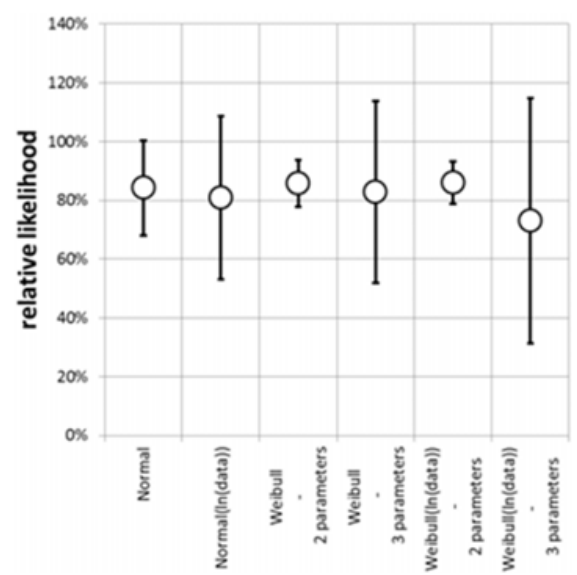

Figure 3. Mean \pm standard deviation of the Relative Likelihood of 6 fatigue strength distributions fitted to the ESC model.

\section{Comparison of the fatigue strength distributions and models}

A goodness-of-fit ranking of the distributions was made by using the relative likelihood method. The 2-p Weibull distribution performed better in terms of median and scatter.

Unfortunately, the relative likelihood can only be used to compare nested models [4]. Qualitative considerations are: 1 - the Extended Stair Case model discards the information of the cycles to failure and so it is believed to be less effective, 2- Castillo-Canteli often seemed to be the less effective in catching the shape of the data and resulted to be the least robust; 3- RFLM usually seemed to perform well although is computationally expensive and not easily modifiable in case one wants to change the distributions; 4- Bi-conditional model has its strength in the flexibility but, similar to RFLM, there is no explicit formulation of useful properties like the iso-probability curve.

\section{References}

[1] Engler-Pinto, C et al., "Statistical Approaches Applied to Fatigue Test Data Analysis," SAE Technical Paper 2005-01-0802 (2005)

[2] Castillo, E. and Canteli, A. F., "A unified statistical methodology for modeling fatigue damage", Springer (2009)

[3] Pascual, F. G. and Meeker, W. Q., "Estimating Fatigue Curves With the Random FatigueLimitModel", Technometrics 41, n. 4 (1999)

[4] Goglio, L; Rossetto, M. "Comparison of Fatigue Data Using Maximum Likelihood Method", Engineering Fracture Mechanics (2004) 